\title{
La gestión del talento humano, recurso indispensable para la organización en el entorno competitivo actual
}

\section{The management of human talent, an indispensable resource for the organization in today's competitive environment}

Lucía Magdalena Pico Versoza

Universidad Internacional del Ecuador, Ecuador

Autor para correspondencia: lupicove@ internacional.edu.ec

Fecha de recepción: 10 de Septiembre de 2016 - Fecha de aceptación: 01 de Noviembre de 2016

\section{Resumen}

Este estudio pretende analizar los modelos de gestión del talento humano, considerando que en la actualidad existen mercados que se encuentran en constante cambio debido a la globalización, lo que evidencia la importancia de contar con empleados capacitados, en donde los verdaderos responsables de la gestión de las organizaciones consideran habilidades, destrezas y competencias de cada trabajador que harán calificarlo como "apto" para formar parte del grupo de empleados que llevarán a la cima a la empresa. Para comprender la forma en que el talento humano llevará a la empresa a la cima anhelada, primero es importante definir ¿Qué es el talento humano?, ¿Cómo debe estar preparado para convertirse en personal calificado? Y analizar los distintos modelos de gestión que proponen los principales autores, considerando que se busca estándares de alto desempeño a través del trabajo en equipo, motivándolos a dar el máximo de sus capacidades y habilidades, logrando la satisfacción de los usuarios con un servicio de calidad y confort, que conlleve a una mutua satisfacción de necesidades entre el empleado y la empresa.

Palabras claves: gestión; talento humano; organización

\begin{abstract}
This study aims to analyze human talent management models, considering there are currently markets that are constantly changing due to globalization, which shows the importance of having trained employees, where the real managers of the management of organizations consider skills and competencies of each worker that will qualify as "fit" to be part of the group of employees that will lead to the top of the company. For understanding how human talent will take the company to the pinnacle, it is important first to define what is human talent? How should you be prepared to become qualified personnel? And analyze the different management models proposed by the main authors, considering that high performance standards are sought through teamwork, motivating them to give their maximum abilities and skills, achieving the satisfaction of users with a quality service, which leads to a mutual satisfaction of needs between the employee and the company.
\end{abstract}

Key words: management; human talent; organization 


\section{Introducción}

Las empresas viven una constante necesidad de adaptarse al mundo globalizado, en donde la más apta sobrevive, lo que la lleva a enfrentarse en un mercado cada vez más competitivo y enfocarse en una correcta administración del talento humano. Estos factores son considerados de mucha importancia al momento de seleccionar el personal idóneo para la empresa, tomando en cuenta ciertos parámetros que deben ser analizados y verificados en relación a su disponibilidad para ocupar los puestos que a su vez cumplan con las necesidades de la empresa.

\section{Contenido}

Chiavenato comenta que el área de recursos humanos $(\mathrm{RH})$, es una de las áreas que más cambios experimenta. Los cambios son tantos y tan grandes que hasta el nombre del área ha cambiado. En muchas organizaciones, la denominación de Administración de Recursos Humanos (ARH) está sustituyéndose por Gestión del Talento Humano. (Chiavenato ,2008)

Por su parte, el control es un proceso que sirve para guiar la gestión empresarial hacia los objetivos de la organización, es decir, el control debe servir de modelo para alcanzar eficazmente los objetivos planteados con el mejor uso de los recursos disponibles (técnicos, humanos, financieros, etc.). Por ello se define como un proceso de retroalimentación de información de uso eficiente de los recursos disponibles de una empresa fin de lograr los objetivos planteados. (Anthony, 2008)

También es importante analizar que el talento humano incluye el esfuerzo de cada trabajador, y la inteligencia de la persona, demostrando sus conocimientos, habilidades, aptitudes, etc. Es así, que se entiende como talento humano una combinación de varios aspectos, características o cualidades de una persona, que implica saber (conocimientos), querer (compromiso) y poder (autoridad).

Los conocimientos corresponden a la sabiduría, inteligencia, razonamientos, etc., mismas que también son conocidas como competencias cognitivas. Los compromisos son las actitudes, temperamento, personalidad y esfuerzo que refleja las competencias personales. En relación al poder tenemos los valores, la decisión y la capacidad personal para hacerlos conocidos como competencias ejecutivas o de liderazgo (Juan Carlos, 2010).

El concepto de talento es congruente con el de competencias, según Martha Alles (2008): "La competencia es una característica de la personalidad devenida en comportamientos que generan un desempeño exitoso en un puesto de trabajo. Las competencias son cualidades que permanecen subyacentes al interior del individuo, las cuales solo se hacen visibles en sus conductas laborales.

El talento humano de una empresa es considerado pieza clave para lograr los objetivos de la misma. Cabe recalcar que ese talento humano debe estar comprometido y motivado para llegar al éxito. En esta área, el talento humano se centraliza en identificar, captar y capacitarse en 
impulsar la productividad de las organizaciones, a la vez que se tiene en cuenta la necesidad de preguntarse sobre la forma en que se está gestionando y la forma en que debería gestionarse.

En la actualidad, muchas empresas se enfrentan a grandes desafíos tales como; empleados desmotivados y con ningún tipo de compromiso con la empresa., lo cual es un punto importante que se debe tener en cuenta al analizar la implementación de técnicas de motivación y así lograr que los empleados se comprometan con las políticas, los objetivos y los procedimientos de la organización.

La motivación es una de las tareas administrativas más simples pero al mismo tiempo una de las más complejas, simplemente por la razón de que las personas se perciben motivadas o impulsadas a comportarse en forma tal que obtengan recompensas, por lo tanto, motivar a un empleado resultaría sencillo si sólo se enfocara en encontrar lo que desea y colocar posibles incentivos, los cuales pueden ser económicos, emocionales o administrativos.

La administración del talento humano en la práctica se efectúa a través del proceso administrativo; planear, ejecutar y controlar. El uso adecuado de los recursos de una empresa, entre ellos el talento humano, no solo representa el esfuerzo o la actividad humana de un grupo de individuos, sino también deben considerarse los factores representativos de estos individuos.

Es un área muy sensible a la mentalidad que predomina en las organizaciones. Es contingente y situacional, pues dependen de aspectos como la cultura de cada organización, la estructura organizacional adoptada, las características del contexto ambiental, el negocio de la organización, la tecnología utilizada, los procesos internos y otra infinidad de variables importantes. Chiavenato (2008).

Para analizar brevemente los modelos de gestión del talento humano se considerarán los conceptos de los principales autores, entre ellos la importancia de analizar que los modelos de gestión representan un nuevo pensamiento metodológico que sirve como impulso hacia la búsqueda de diferentes modelos conceptuales. La mayoría de modelos de gestión trabajan con sistemas informáticos e implementos tecnológicos que han elevado a un nivel más alto la eficacia de los recursos humanos en la empresa, posicionándolos en un lugar fundamental para que la empresa pueda lograr concretamente cada uno de los objetivos planteados.

Los modelos de gestión son muy diferentes entre sí, pero es importante destacar que todos y cada uno de ellos poseen niveles de eficiencia que deben aplicarse según el tipo de empresa en la que se practica la gestión correspondiente. Estos elementos se van integrando en la gestión empresarial, obligando al modelo de negocio tradicional a evolucionar hacia nuevos modelos de gestión mejorando así la competitividad y los resultados empresariales.

Los nuevos preceptos acerca del factor humano y hacia la GRH han sido incorporados en mayor o menor medida de acuerdo a cada organización, y se ha desencadenado la necesidad de contar con un sistema en la administración de los recursos humanos, adecuado a los requerimientos organizacionales, así lo plantean Velásquez y Miguel (2001) cuando sostiene que gracias al productos de factores socioeconómicos condicionantes, se ha llevado a la concepción de diferentes modelos para la aplicación práctica de sistemas de gestión de estos recursos. 
Entre los modelos clásicos, junto con otros contemporáneos, partes del análisis se precisa que cada uno de estos modelos exige un nuevo tratamiento la dirección de recursos humanos reconociendo su valor indiscutible y el papel que juega en el desarrollo de la empresa. Cada modelo posee ventajas y desventajas por tanto se debe analizar la situación específica de la empresa y del entorno para que se adopte el más adecuado a la realidad de la misma.

\section{Modelo de Harper y Lynch}

El modelo de Harper y Lynch (1992) indica que a partir del plan estratégico se realiza la previsión de necesidades en interdependencia con una serie de actividades claves de la dirección de recursos humanos (análisis y descripción de puestos, curvas profesionales, promoción, planes de sucesión, formación, clima y motivación, etc.) desarrolladas a través del conocimiento de la dirección de recursos humanos, con que cuenta la organización. Con estos elementos se lograra una optimización de los recursos humanos, para lo cual se requiere de un seguimiento constante verificando la coincidencia entre los resultados obtenidos y las exigencias de la organización.

Tiene carácter descriptivo, solo muestra las actividades relacionadas con la GRH para lograr su optimización, pero no en su dinámica y operación.

El modelo determina en que cantidad y calidad, precisamente permita satisfacer la demanda, mediante la realización de un conjunto de actividades que se inician con el inventario de personal y la evaluación del potencial humano. Siendo este un análisis de las políticas y prácticas del personal de una organización y evaluación de su funcionamiento actual, teniendo por objetivos llegar a una opción profesional sobre las acciones llevadas a cabo en materia de recursos humanos. (Ballivian D. Rafael A, 2006).

\section{Modelo de Wherter y Davis}

En este modelo se plantea la interdependencia entre las actividades de recursos humanos, que son agrupadas en cinco categorías y hoy son trascendentes en la GRH así como los objetivos sociales, organizacionales, funcionales y personales que rigen en la empresa.

Sin embargo, este modelo no cuenta con una proyección estratégica de los recursos humanos, pero es positivo el papel inicial que le otorga a los fundamentos y desafíos, donde incluye al entorno como base para establecer el sistema y muestra a la autoridad como elemento de retroalimentación y de continuidad en la operación de la GRH.

Werther y Davis señalan cuatro tipos de objetivos del departamento de recursos humanos, los cuales son corporativos, funcionales, sociales y personales. (Ballivian D. Rafael A, 2006)

\section{Modelo de Zayas}

Existe también el modelo de Zayas (1996), en el que se destaca el carácter sistemático de la GRH, planeado una interdependencia entre los tres subsistemas de organización; el de 
selección y desarrollo de personal; y el formado por el hombre y las distintas interacciones que el mismo establece.

A partir de la determinación de la misión, se definen los objetivos y la estructura organizativa y de dirección, lo que lleva implícito el diseño de cargos y ocupaciones, determinando así las exigencias y requerimientos de los mismos y las características que deben poseer los trabajadores.

Todo ello servirá de base para definir las fuentes de reclutamiento, los métodos de selección y la formación y desarrollo del personal, lo que condiciona las características del personal que ingresa en la organización y las interrelaciones que se producen. (Ballivian D. Rafael A,2006)

\section{Modelo de Idalberto Chiavenato}

En el modelo Idalberto Chiavenato, se plantea que los principales procesos de la moderna gestión del talento humano se centran en seis vertientes tales como la admisión de personas, relacionado directamente con el reclutamiento y selección, en la aplicación de personas (diseño y evaluación del desempeño), en la compensación laboral, en el desarrollo de las personas y en el monitoreo de las personas basados en sistemas de información y base de datos. Estos procesos están influenciados por las condiciones externas de la organización. (Ballivian D. Rafael A, 2006),

Chiavenato señala que el reclutamiento consiste en un conjunto de procedimientos que tienden a atraer candidatos potencialmente calificados y capaces de ocupar cargos dentro de la organización. Básicamente es un sistema de información, mediante el cual la organización divulga y ofrece al mercado de talento humano oportunidades de empleo.

En relación a la selección del personal, el autor indica que es el proceso mediante el cual se busca entre los candidatos reclutados a los más adecuados para cubrir las vacantes en la empresa, con el fin de conservar o ampliar la eficiencia y el desempeño personal, que incidirá directamente en la eficiencia de la organización. El proceso de selección proporciona mayor objetividad y precisión, siendo así la selección del personal una importante tarea en la forma de un proceso de comparación y decisión, que tiene su punto de partida en el diseño de cargo.

Para Chiavenato, la comprobación de los conocimientos y experiencias de los candidatos, así como la valoración de sus habilidades, potenciales y características de su personalidad consisten en técnicas de selección, entrevistas, pruebas de personalidad y técnicas de simulación. Una buena técnica de selección debe dar el mejor pronóstico sobre el desempeño futuro del candidato.

Este modelo también contempla la aplicación de personas, división de cargos y salarios, inducción y entrenamiento de personal. Es así, que el proceso de inducción provee al candidato de herramientas que facilitan la adaptación y socialización con la cultura de la organización, las normas que rigen la organización a fin de proporcionar al nuevo empleado, relaciones de 
desempeño que contribuyan al éxito en la organización. El objetivo principal de esta etapa es brindar al trabajador una efectiva orientación sobre su puesto y funciones que debe desempeñar.

Luego de la inducción, la organización se ocupa del entrenamiento, cuyo objetivo primordial es abrir el camino al nuevo empleado en forma rápida y económica, mejorando la cantidad y calidad de trabajo, equipando al nuevo empleado con los conocimientos necesarios.

Las ventajas del entrenamiento son incrementar la eficiencia, motivas al personal, elevar el compromiso del empleado, mejorar organización e incremento de las utilidades como consecuencia de la eficiencia.

Chiavenato sugiere también considerar la compensación de las personas y división de beneficios sociales. De acuerdo con Morales (1999), se entiende por compensación el pago total que hace la organización al trabajador por labor contratada, que incluye beneficios, prestaciones e incentivos o remuneraciones por rendimiento.

Los programas de compensación benefician la base económica del empleado y su familia, le dan la posibilidad de ganancias elevadas, lo cual motiva al empleado, debido a que eleva su autoestima.

En cuanto a la división de capacitación, se sugiere que la misma esté dirigida para el perfeccionamiento técnico del trabajador para que este se desempeñe eficientemente en las funciones que se le asigna. Chiavenato (2002) apunta que mediante las jornadas de capacitación se pueden actualizar conocimientos y perfeccionar las habilidades adquirida por los empleados, creando una actitud positiva, identificando al personal con los objetivos de la empresa.

El proceso de evaluación de desempeño también resulta fundamental, ya que ofrece a la gerencia de talento humano la oportunidad de trabajar sobre el desempeño de los empleados en el corto plazo y sobre su desarrollo. Este proceso de evaluación de desempeño permite dar solución a problemas de integración del tajador a la organización o al cargo que ocupa actualmente, de la falta de aprovechamiento de potenciales mayores que los exigidos para el cargo. Así como también, lograr una mejor comunicación y planificar y organizar el trabajo en fortalezas y debilidades detectadas, constituye para la organización un recurso válido para el aumento de sueldo por mérito, asignación de gratificaciones y despidos.

A continuación se propone argumentos que se considera justifican dentro del proceso de evaluación de desempeño de los trabajadores:

- Proporciona un juicio sistemático para fundamentar aumentos salariales, promociones, transferencias y despido de empleados.

- Permite comunicar a los empleados como marchan en el trabajo, que deben cambiar en el comportamiento, en las actitudes, las habilidades o los conocimientos.

- Posibilita que los subordinados conozcan lo que el jefe piensa de ellos.

La evaluación de desempeño debe proporcionar beneficios a la organización y a las personas; en consecuencia. Se debe tener en cuenta las siguientes líneas básicas: 
- La evaluación no debe abarca no solo el desempeño en el cargo ocupado, sino por el contrario del alcance de metas y objetivos.

- La evaluación debe hacer énfasis en el individuo que ocupa el cargo y no en la impresión resto de los hábitos personales observados en el trabajo.

- La evaluación debe ser aceptada por ambas partes; evaluador y evaluado. Ambos deben estar de acuerdo en que la evaluación debe traer algún beneficio para la organización y para el empleado.

\section{Modelo de Beer y colaboradores}

Estos autores abarcan todas las actividades claves de la GRH en cuatro áreas con las mismas denominaciones de estas políticas.

Se explica que la influencia de los empleados lo que respecta la participación, y el involucramiento se considera central, actuando sobre las restantes aéreas o políticas del departamento de talento humano. (Ballivian D. Rafael A.,2006)

\section{Modelo de GRH de diagnóstico, proyección y control (DPC). (Cuesta, 2005)}

A partir del modelo de Beer, modificado por Cuesta y otros elementos, surge este modelo de GRH funcional, que también es concebido por Cuesta, e implica la incorporación de tecnología para llevarlo a la práctica organizacional. En el que quedan concentradas todas las actividades clave de GRH en los cuatro subsistemas de GRH:

- Flujo de recursos humanos: inventario de personal, selección de personal, colación, evaluación del desempeño, evaluación del potencial humano.

- Educación y desarrollo: formación, planes de carrera, planes de comunicación.

- Sistemas de trabajo: organización de trabajo: organización de trabajo, seguridad e higiene ocupacional.

- Compensación laboral: sistemas de pago, sistemas de reconocimiento social, sistemas de motivación.

\section{Conclusiones}

A partir de los modelos de gestión del talento humano analizados, se considera que los empleados constituyen el principal activo de la organización. La gestión del talento humano en las organizaciones es la función que permite la colaboración eficaz de las personas, para alcanzar los objetivos organizacionales e individuadles.

Cada modelo analizado presenta opciones que la GRH puede optar por analizar en función del bienestar de la empresa, precisando que se contribuya a la eficacia organizacional a través de los siguientes medios:

- Ayudar a la organización a alcanzar los objetivos y realizar su misión.

- Proporcionar competitividad a la organización.

- Suministrar a la organización empleados bien entrenados y motivados.

- Permitir el aumento de la autorrealización y la satisfacción de los empleados en el trabajo.

- Desarrollar y mantener la calidad de vida en el trabajo. 
- Administrar el cambio.

- Establecer políticas éticas desarrollar comportamientos socialmente responsables.

Resulta imprescindible considerar que los distintos modelos analizados buscan generar talento humano apto y capacitado de acuerdo a los requerimientos de las empresas, en donde a la vez se identifica al autor Idalberto Chiavenato con un modelo que prevalece en el proceso de gestión que incluye admisión de personas, aplicación de personas, comprensión de personas, desarrollo de personas, mantenimiento de personas y monitoreo de personas, siendo este el modelo que actualmente gana más terreno en el ámbito empresarial.

Finalmente, prevalece la propuesta de mejoramiento de la gestión del talento humano,, la cual es indispensable para la empresa que pretende asignar procesos de reclutamiento y selección de personal a un departamento específico, cambiando la actitud del personal implementando la comunicación entre todos los que conforman la empresa, creando un clima organizacional favorable en bienestar de los trabajadores y la empresa.

\section{Bibliografía}

Bautista, V. y. (2003). Modelos de gestión del talento humano. Obtenido de www.gestiopolis.com

Cabrera, R. y. (1999). Las cuatro caras de la Dirección de Recursos Humanos.

Chiavenato, I. (1999). Administración de recursos humanos. México: Ed. McGraw-Hill.

Chiaventato, I. (2002). Gestión del talento humano. Bogotá: Ed. Pretice Hall.

Cuesta, A. (2005). Tecnología de gestión de recursos humanos. La Habana: Ed. Academia.

Werther, W. y. (2001). Administración de personal y recursos humanos, 5ta. edición. México: Ed. Mc. Graw Hill.

Chiavenato, I. (2006). Introducción a la teoría general de la administración. México: Mc Graw Hill.

Chiavenato, I. (2011). Administración de recursos humanos. Bogotá: Mc Graw Hill.

Kiernan, M. (2003). Once mandamientos de la gerencia del siglo XXI. Ed Prentice Hall.

Reyes, A. (2005). Administración de personal. México: Limusa.

Sastre, M. y. (2003). Dirección de recursos humanos, Remuneraciones. Madrid: Mc Graw Hill.

Werther, W. (2008). Administración de recursos humanos. Bogotá: Mc Graw Hill.

Wether, W. y. (2008). Administración del personal y recursos humanos. México: Mc Graw Hill. 